\title{
Cognitive and Neural Aspects in Robotics with Applications 2011
}

\author{
Madan M. Gupta, ${ }^{1}$ Ivo Bukovsky, ${ }^{2}$ Noriyasu Homma, ${ }^{3}$ Zeng-Guang Hou, ${ }^{4}$ \\ and Ashu M. G. Solo ${ }^{5}$ \\ ${ }^{1}$ Intelligent Systems Research Laboratory, College of Engineering, University of Saskatchewan, Saskatoon, SK, Canada S7N 5A9 \\ ${ }^{2}$ Division of Automatic Control and Engineering Informatics, Department of Instrumentation and Control Engineering, \\ Faculty of Mechanical Engineering, Czech Technical University in Prague, Technicka 4, Prague, 16607, Czech Republic \\ ${ }^{3}$ Cyberscience Center, Tohoku University, 6-3 Aoba, Aramaki, Aoba-Ku, Sendai 980-8578, Japan \\ ${ }^{4}$ Institute of Automation, The Chinese Academy of Sciences, P.O. Box 2728, Beijing 100190, China \\ ${ }^{5}$ Maverick Technologies America Inc., Suite 808, 1220 North Market Street, Wilmington, DE 19801, USA
}

Correspondence should be addressed to Madan M. Gupta, madan.gupta@usask.ca

Received 19 January 2012; Accepted 19 January 2012

Copyright (C) 2012 Madan M. Gupta et al. This is an open access article distributed under the Creative Commons Attribution License, which permits unrestricted use, distribution, and reproduction in any medium, provided the original work is properly cited.

With the evolution of our complex technological society and the introduction of new notions and innovative theoretical tools such as cognitive and neural aspects of robotics, there have been some new evolutions of theoretical methodology. These evolving and innovative theoretical tools are providing some intelligence and robustness in robotic systems similar to what is found in natural biological species.

Cognition and intelligence- - the ability to learn, understand, and adapt - are the creation of nature, and they play a key role in human actions as well as in many other biological species. Humans possess some robust attributes of learning and adaptation and that is what makes them so intelligent.

Humans react through the process of learning and adaptation on information received through a widely distributed network of sensors and control mechanisms in our body. The faculty of cognition which is contained in our carbon-based computer-the brain-acquires information from the environment through various sensory mechanisms such as vision, hearing, touch, taste, and smell. Then the process of cognition-cognitive computing-integrates this information through its intricate neural networks and provides appropriate actions. The cognitive process then advances further toward some attributes such as learning, recollection, reasoning, and control.

We are learning from the carbon-based cognitive computer-the brain-and are now in the process of inducing perception, cognition, and intelligence in robotics machines. One of our aims is to construct a robotic vehicle that can think and operate in uncertain and unstructured driving conditions. Robots in manufacturing, mining, agriculture, space and ocean exploration, and health sciences are just a few examples of challenging applications where human attributes such as cognition and intelligence can play an important role.

The proposal for this second special issue on cognitive and neural aspects of robotics with applications was conceived in late 2010, and we are now pleased to present 8 research papers that cover a wide aspect of cognition and intelligence. Initially, we received 15 research papers, but after going through a thorough review process, we have accepted only 8 research papers. These 8 accepted research papers cover some wider aspects of cognition and intelligence in the field of robotics, and for this special issue, we have divided these 8 research papers into three groups.

Four research papers cover the fields of cognition, perception, and neural learning in robotics. In the research paper entitled "Control loop sensor calibration using neural networks for robotic control," Kathleen A. Kramer and Stephen C. Stubberud present a technique referred to as a neural extended Kalman filter (NEKF) to provide both state estimation in a control loop and learn the difference between true sensor dynamics and the sensor model. The resulting sensor model provides better estimation capability and redundancy. In the research paper entitled "3D Assembly 
group analysis for cognitive automation," Christian Brecher, Thomas Breitbach, Simon Müller, Marcel Ph. Mayer, Barbara Odenthal, Christopher Schlick, and Werner Herfs introduce a novel concept that allows for the cognitive automation of robotic assembly processes and use an assembly cell of two robots to verify the concept. In the research paper entitled "Optimal search strategy of robotic assembly based on neural vibration learning," Lejla Banjanovic-Mehmedovic, Senad Karic, and Fahrudin Mehmedovic present the implementation of an optimal search strategy in verification of a robotic assembly process based on neural vibration learning. In the research paper entitled "A cognitive model for generalization during sequential learning," Ashish Gupta, Lovekesh Vig, and David C. Noelle present a detailed analysis of the Leabra cognitive modeling framework for sequential learning of multiple tasks because traditional neural network models are trained for one task, and when they are trained to do a new task, they forget how to do the original task. Then they demonstrate the applicability of sequential learning to a pair of movement tasks using a simulated robotic arm.

Two research papers deal with neurocontrol and neural architecture in robotics. In the research paper entitled "Discovering and characterizing hidden variables using a novel neural network architecture (LO-net)," Soumi Ray and Tim Oates present a novel neural network architecture for solving problems related to theoretical entities, which are aspects of the world that cannot be sensed but are causally relevant, such as black holes and dark matter. Their novel neural network architecture discovers that theoretical entities exist, computes their number, and computes their values. In the research paper entitled "Design of an errorbased adaptive controller for a flexible robot arm using dynamic pole motion approach," Ki-Yong Song, Madan M. Gupta, and Noriyasu Homma introduce a novel concept of dynamic pole motion (DPM) for the design of an errorbased adaptive controller (E-BAC). The purpose of this novel design approach is to make the system response reasonably fast with no overshoot where the system may be time varying and nonlinear with only partially known dynamics. For illustrating the strength of this novel design approach, they give an example of a flexible robot with nonlinear dynamics.

Finally, two research papers focus on miscellaneous robotics applications including underwater robotics and the development of bio-machine. In the research paper entitled "Application of on-board evolutionary algorithms to underwater robots to optimally replan missions with energy constraints," Mae L. Seto presents an on-board knowledge-based agent based on a genetic algorithm to replan a near optimal survey mission for an autonomous underwater vehicle (AUV) given the AUV energy budget, mission duration, and survey area dimensions. Finally, in the research paper entitled "Development of bio-machine based on the plant response to external stimuli," Aditya. K., Ganesha Udupa, and Yongkwun Lee present research on plant intelligence. Specifically, they present research on plant action potential signals and their response to various light modes for control of a biomachine.

Thus, as can be seen, these 8 research papers represent a broad cross-section of cognitive and neural aspects of the field of robotics. Also, the authors of these research papers have used some cognitive and neural aspects of robotics in the design of learning and control algorithms. These research papers have been authored or coauthored by 24 researchers in the robotics field from 14 different research institutions or universities located in 7 different countries: Bosnia and Herzegovina, Canada, China, India, Japan, Korea, and U.S.A.

This second special issue including 8 contributed research papers, which are authored by international researchers and devoted to various aspects of cognition, perception, neural learning, and neurocontrol in robotics with various industrial applications, is an informative and useful addition to the field of robotics.

\section{Acknowledgments}

We, the guest editors, would like to express our sincere appreciation to the editorial board of the Journal of Robotics and Hindawi for their confidence and unwavering support for this second special issue. We acknowledge the efforts of the authors for their valuable research contributions to this special issue of the journal and that of the reviewers who adhered to the strict timeline for making this special issue a success.

Madan M. Gupta Ivo Bukovsky Noriyasu Homma Zeng-Guang Hou Ashu M. G. Solo 

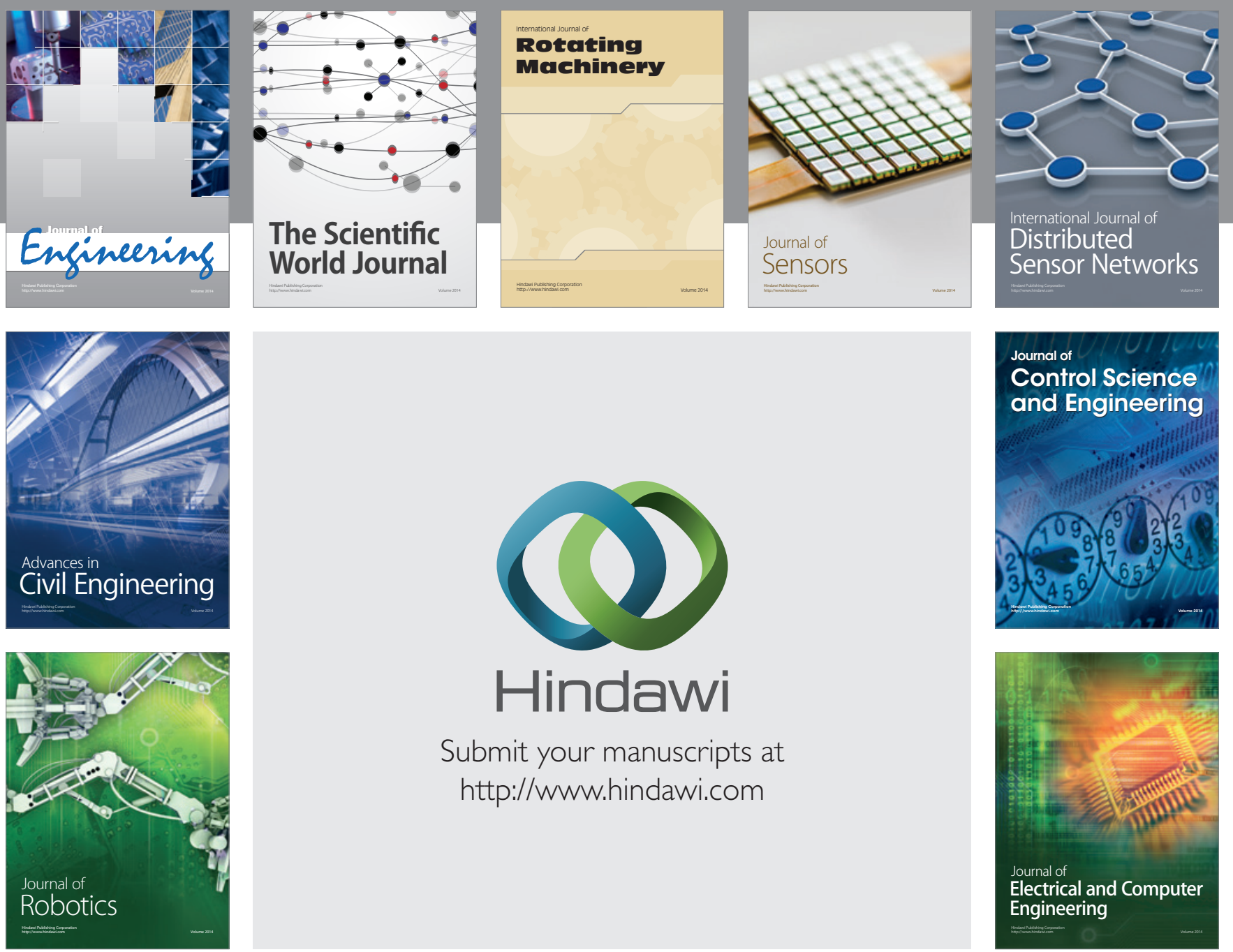

Submit your manuscripts at

http://www.hindawi.com
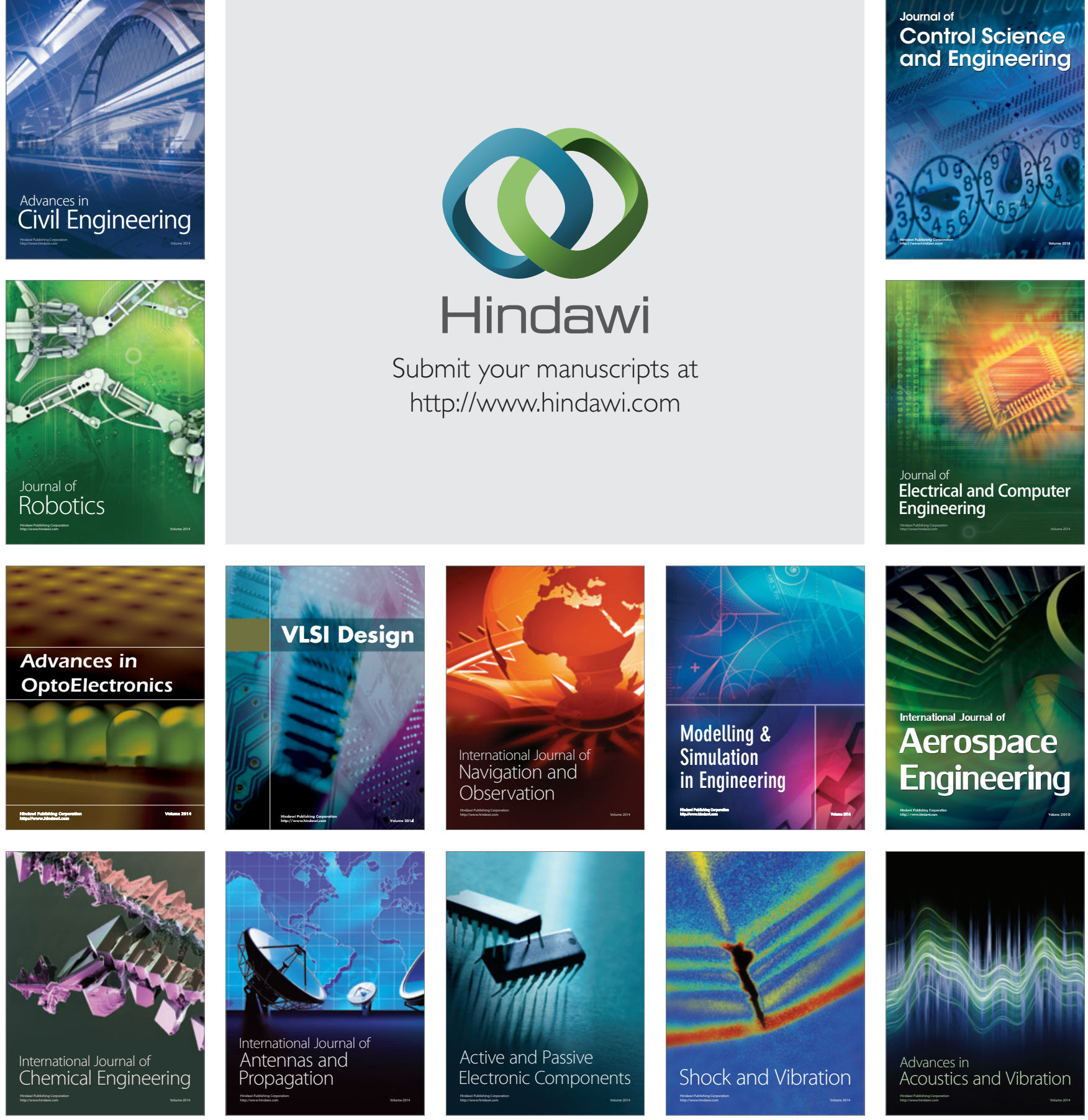